\title{
Nano-Molecular Junctions on STM Tips
}

\author{
Chun Huang*, Jianshu Yang
}

(Received 14 Nov. 2010; accepted 16 Mar. 2011; published online 31 March 2011.)

\begin{abstract}
We present a technique for building metal-organic-metal junctions, which contain ten or fewer conjugated molecules between each of such junction, and the investigations of the I- $\mathrm{V}$ response of these junctions. The junctions are made by self assembling thiolated molecules onto gold coated tips for use in scanning tunneling microscopy. We show that this easy technique probes the qualitative properties of the molecules. Currentvoltage characteristics of a Tour wire and a new molecular rectifier are presented.
\end{abstract}

Keywords: STM; Junction; I-V response; Molecules; Tour wire

Citation: Chun Huang and Jianshu Yang, "Nano-Molecular Junctions on STM Tips", Nano-Micro Lett. 3 (1), 1-5 (2011). http://dx.doi.org/10.3786/nml.v3i1.p1-5

Since the use of molecules for electronics was first described theoretically in the 1970s [1], numerous experiments have been conducted with scanning tunneling microscopy (STM), [2] conducting atomic force microscopy (AFM) [3] and the break junction [4] to probe their electronic properties. Molecules, connected to two metallic electrodes, can act as diodes, configurable memory devices, single-molecule rectifiers, transistors, or negative differential resistors [5].

Electron transport through the molecular junction is determined not only by the intrinsic electronic structure of the molecule but also by the metal-molecule contacts [6-9]. A good electrical contact between a molecule and a conducting substrate is a chemical bond, usually achieved with sulfur or selenium bound to gold [10]. In principle it is easy to make use of this concept to self assemble molecules at one electrode and make molecular junctions with a scanning probe tip. For instance, transport through single or at most a few molecules on a gold surface was observed with STM [11] using the tip as a counter electrode. Cui et al. [12] used thiol groups to bond to a gold based electrode and a gold nanoparticle top electrode. Reichert et al. [13] introduced small molecules between the electrodes in a mechanically controllable break junctions. Zhitenev et al. [14] made contact to the molecule with a gold-coated AFM tip, so that the molecule was covalently bonded to gold at both ends.

In this work, we investigated an easy technique for fabricating and studying molecular nanojunctions using an STM. Compared to conventional molecule junctions realized in STM measurements, we adopted inverse junction geometry by assembling molecules onto a gold-coated tungsten STM tip, which allowed us to investigate the I-V curves at room temperature. This is actually challenging for other normal methods $[2,3]$. The tip was brought into contact with a clean gold surface and the current-voltage (I-V) characteristics were measured. A schematic of this configuration is shown in Fig. 1(a). We present results on I-V characteristics of two different molecules, shown in Fig. 1(b), named as 4, 4'-bis(phenylethynyl)benzenethiolate (BPEBT) and 4-nonafluorobiphenylbenzenethiolate (FFBT). Here, BPEBT could be considered as a molecular wire while FFBT is a diode-like molecule due to the electron withdrawing effect of fluorine atoms.

As shown below, reproducible I-V curves for the BPEBT wire and asymmetric I-V indicating rectifying behavior for FFBT were obtained at room temperature. This shows the utility of the inverse molecule junction technique for scanning tunneling spectroscopy (STS) measurement of organic molecules, in which the local measurements of the tunneling current versus tip-

Institute of Materials Research and Engineering, 3 Research Link, Singapore 117602

*Corresponding author. E-mail: huangchun0713@hotmail.com 
sample bias (I-V) curve were performed with a certain tip to substrate distance. We found the I-V characteristics, obtained by probing a self assembled monolayer of the same molecules on a gold substrate by a bare STM tip had the same form as the data shown below, but exhibited significantly higher noise. It should be emphasized at the outset that the method is only suited to qualitative assessment of the molecule I-V characteristics because the number of molecules in the junction and the pressures acting on the molecules [15] are not known. Therefore, we consider this technique as a quick method for testing the general $\mathrm{I}-\mathrm{V}$ behavior of a number of molecules.

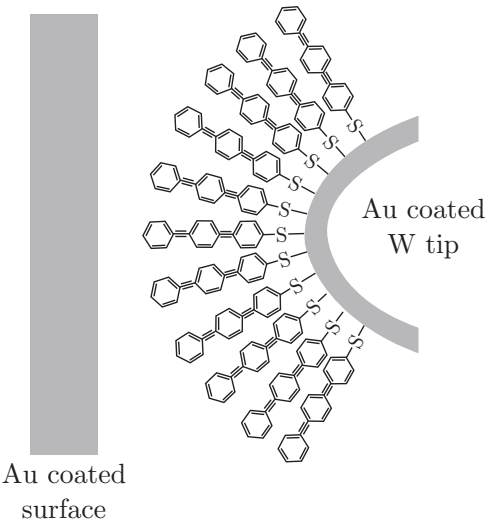

(a)

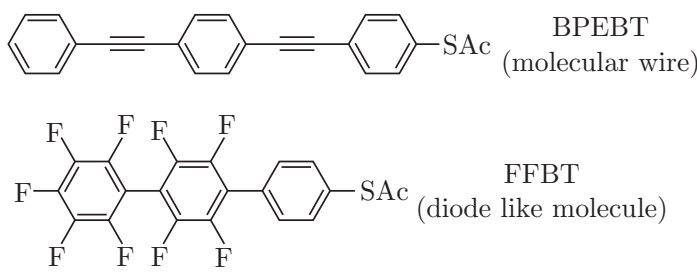

(b)

Fig. 1 (a) Schematic of the junction geometry used. Molecules self assembled onto a gold coated STM tip are brought up to a $\mathrm{Au}(111)$ surface. (b) Schematic structures of the molecules investigated. BPEBT is a molecular wire. FFBT is a molecular diode.

The experiments were carried out in an Omicron MULTPROBE S ultrahigh vacuum (UHV) STM system with a base pressure higher than $8 \times 10^{-9} \mathrm{~Pa}$. The substrate used was gold (111) film deposited by thermal evaporation onto freshly cleaved mica. Prior to deposition the mica was degassed for $6 \mathrm{~h}$ at $450{ }^{\circ} \mathrm{C}$ in vacuum. The gold deposition rate was $0.1 \mathrm{~nm} / \mathrm{s}$ and the thickness of gold film was typically $70 \mathrm{~nm}$. After the film deposition, the sample was kept in the vacuum chamber for another $30 \mathrm{~min}$ at $500^{\circ} \mathrm{C}$ for annealing of the surface.

The STM tip was made by chemical etching of a $0.25 \mathrm{~mm}$ diameter tungsten wire in $2 \mathrm{M} \mathrm{NaOH}$. Freshly etched tungsten tips were coated with gold using the same methodology described above. The two types of molecule (BPEBT and FFBT) were then chemically bonded to the tips. All molecules consist of a conjugated rigid backbone with thiol groups on one end to form stable covalent bonds to gold electrodes. The length of the molecules is around $2 \mathrm{~nm}$. Self-assembled films on the tip were obtained by immersing the goldcoated tips in 1mM BPEBT or FFBT in tetrahydrofuran (THF) solution, with a drop of ammonium hydroxide added to displace the acetyl group [16]. The tips were left in solution for around 21 hours, after which they were thoroughly rinsed with THF and then ethanol to remove any physisorbed materials. The prepared tips were immediately introduced into the UHV STM chamber. An atomically flat area of the sample without defects and steps was chosen for experimentation.

Figure 2 shows IV curves obtained in UHV at room temperature for the Tour wire. The I-V curves are consistent with the published literature, with the current at $0.5 \mathrm{~V}$ being $10 \mathrm{pA}$, the approximate value reported in the literature for a Tour wire [17]. Therefore, we can be confident that very few molecules are being probed. Since the HOMO and LUMO for BPEBT are 5.06 and $2.72 \mathrm{eV}$ respectively, and the work function of $\mathrm{Au}$ is $5.1 \mathrm{eV}$, there is good alignment of the Fermi level with the HOMO of the molecule. Therefore the BPEBT molecule should act as a simple wire and exhibit no strong IV asymmetry [18-21]. A slight asymmetry in the data of Fig. 2 is attributable to the small asymmetry in the barriers at the two ends of the molecule, i.e. one end is thiolated and bonded to the gold coated STM tip and the other (un-thiolated) end is in proximity to the gold substrate. A question is naturally arisen as to whether a vacuum tunneling gap exists between the molecule and the gold substrate. Current-distance (I-Z) curves show that the molecule is in contact with the gold substrate, as discussed below for FFBT.

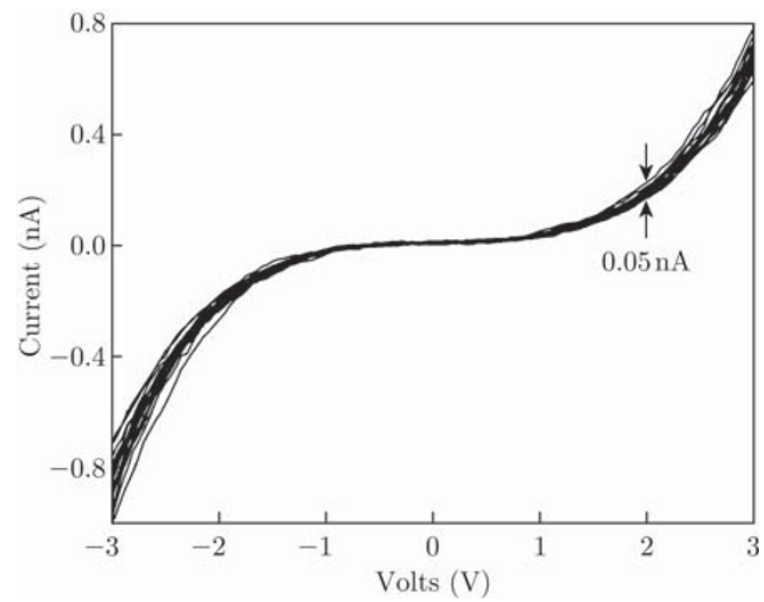

Fig. 2 I-V characteristics of the Tour wire BPEBT, taken at STM conditions of sample bias $\mathrm{V}_{\mathrm{s}}=3.0 \mathrm{~V}$ and tunnel current $I_{t}=0.6 \mathrm{nA}$. The data shows 19 superimposed IV curves with the spread in data being around $0.05 \mathrm{nA}$ at $+2 \mathrm{~V}$. The voltage axis is the sample bias. 
Data for the FFBT molecule is shown in Fig. 3 and 4. Figure 3(a) shows the most common current-distance (I-Z) curves observed for FFBT with an exponential dependence of the current on the tip sample separation. Less common ones were quasi-linear I-Z curves (e.g. Fig. 3(b)) in which abrupt changes in current are observed, presumably arising from changing tip conditions. The abrupt changes may reflect a cross over to mechanical contact but this cannot be verified. In both cases a large displacement is needed ( 1 to $2 \mathrm{~nm}$ ) to change the current from 0.2 to $30 \mathrm{nA}$. Given that the molecules are $\sim 2 \mathrm{~nm}$ in length, one expects that if the indentation is too large, the molecules will be grossly influenced by the applied pressures. This is supported
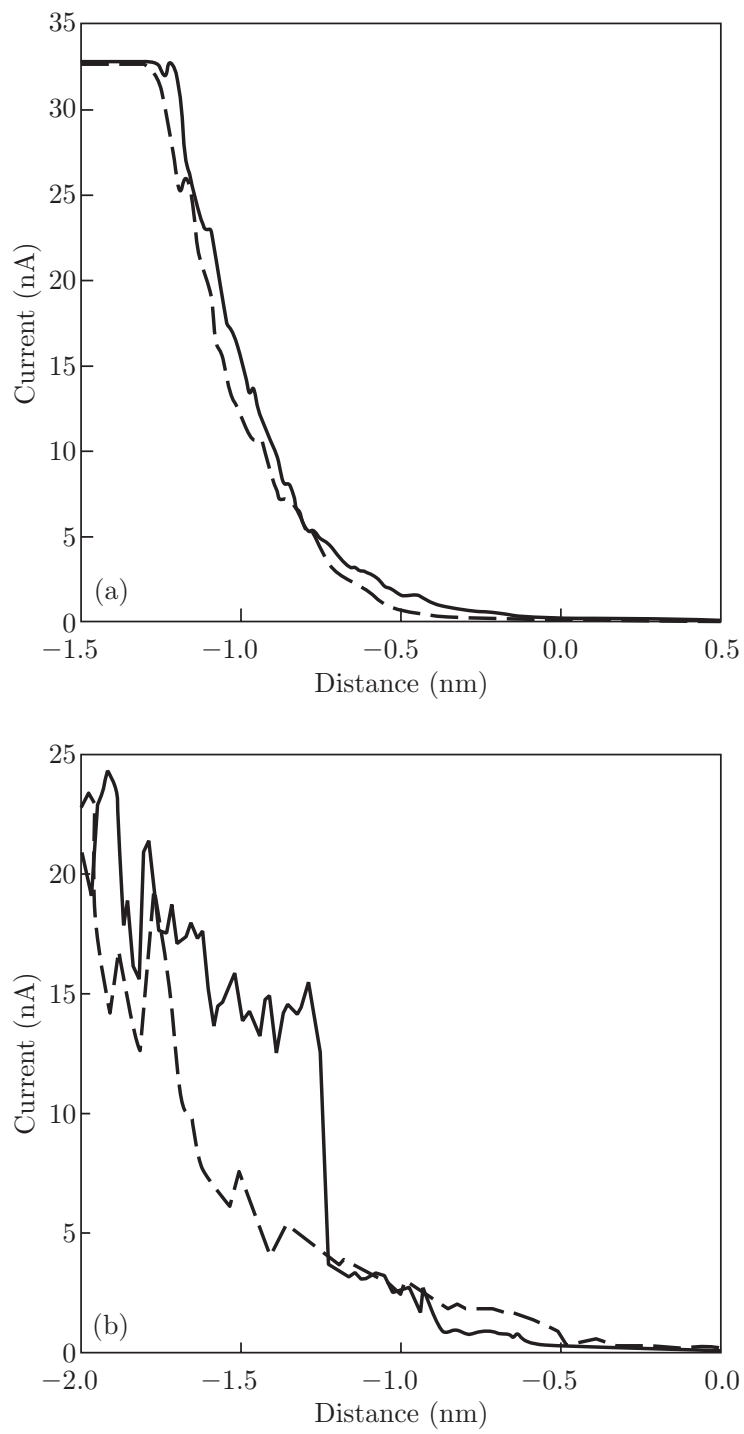

Fig. 3 Current versus relative tip-sample distance (I-Z curve) for the FFBT molecule. The STM control condition, which equates to the relative distance of $\mathrm{Z}=0 \mathrm{~nm}$, is with sample bias $\mathrm{V}_{\mathrm{s}}=2.6 \mathrm{~V}$ and tunnel current $\mathrm{I}_{\mathrm{t}}=0.2 \mathrm{nA}$. The current amplifier saturates at $33 \mathrm{nA}$.
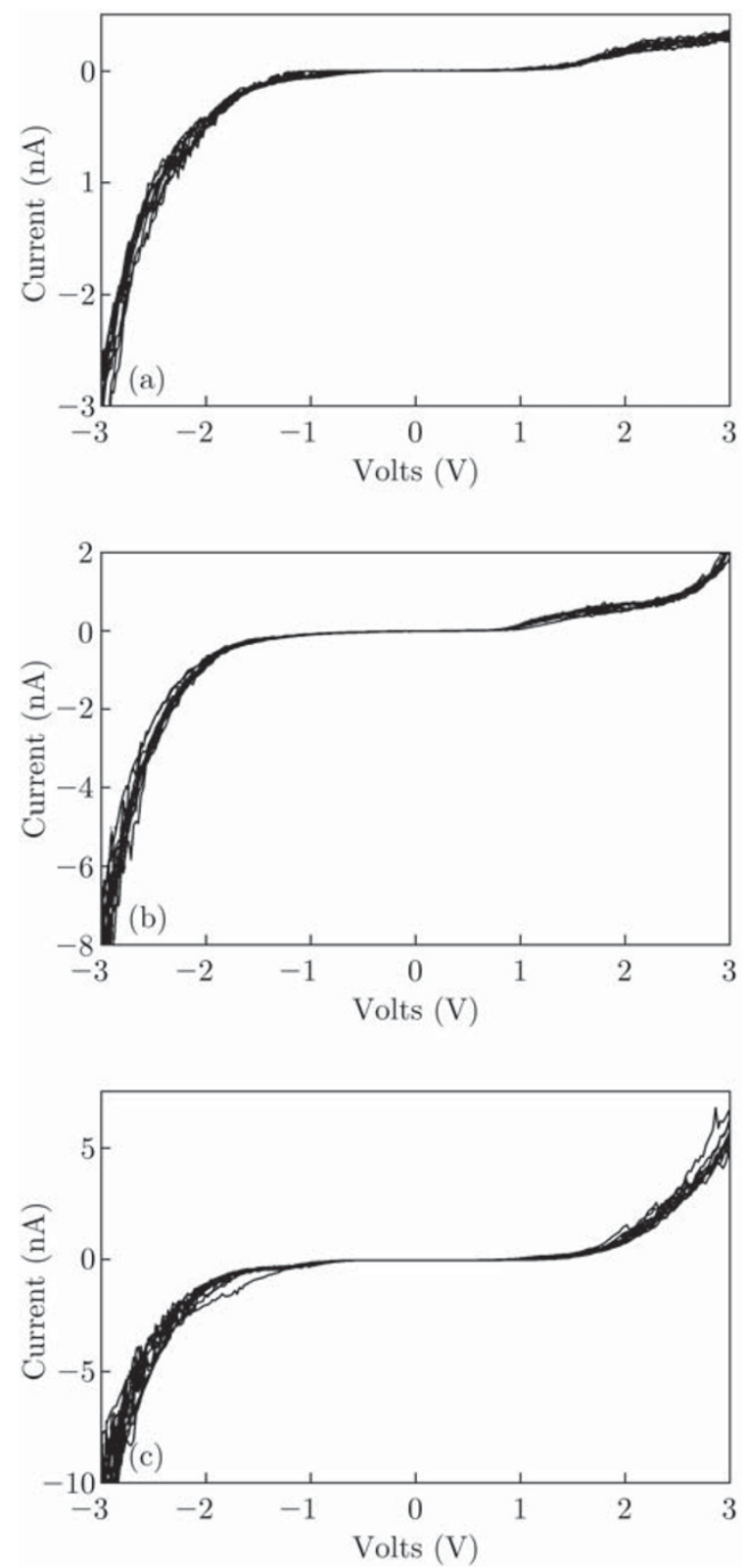

Fig. 4 (a)-(c) I-V curves for the FFBT molecule taken at different STM control current $\left(\mathrm{I}_{\mathrm{t}}=0.4,1.6,4.4 \mathrm{nA}\right)$ with sample bias $V_{s}=2.6 \mathrm{~V}$. The $\mathrm{STM}$ control conditions correspond to different tip-sample separation (see Fig. 3). The voltage axis is the sample bias.

by the I-V data which shows considerable variability if the tunnel current control value $\left(\mathrm{I}_{\mathrm{t}}\right)$ is set larger than $\mathrm{I}_{\mathrm{t}} \sim 10 \mathrm{nA}$.

We conclude from studying series of I-V and I-Z data that for consistent IV data the I-Z curve should be reproducible (no abrupt changes) to ensure a reproducible tip condition and the I-V curve should be taken at only moderate indentation to ensure a good mechanical contact with no vacuum gap and minimal tip damage. The best indentation will depend on the molecule under test. For the FFBT and BPEBT molecules the preferred indentation for $\mathrm{I}-\mathrm{V}$ measurement appears to be 
$\mathrm{I}_{\mathrm{t}} \sim 5 \mathrm{nA}$, whereas at control currents $\mathrm{I}_{\mathrm{t}}=0$ to $\sim 2 \mathrm{nA}$ there is a vacuum barrier or weak mechanical contact (as discussed below for the IV data) and for $I_{t}>10 n A$ the mechanical contact is excessive.

Figure 4 shows three sets of I-V curves of the molecule FFBT which is expected to exhibit diode properties [22-24] in the absence of conformation change. The spectra are taken at different tip-sample separations on the corresponding I-Z curve of Fig. 3. We first discuss Fig. 4(a) and 4(b) which show strong rectification. The I-V curves shown in Fig. 4(a) and 4(b) fit the exponential functional form expected for a vacuum tunnel barrier and we conclude that a vacuum tunnel gap is present. The asymmetric I-V nature in this case arises because the charge injection barrier will be different for either positive or negative sample polarity. In addition, there is a vacuum tunneling barrier at one end of the molecule.

In contrast, we find that the I-V curve for FFBT in Fig. 4(c) is non-linear and a power-law dependence on the voltage provides a best fit. The power law dependence of the current on the voltage in the I-V and the I-Z data of Fig. 3 suggest that the molecule is in good contact with the gold substrate. The I-V data again shows rectification, although with a smaller rectification ratio than in Fig. 4(a) and 4(b). Since the electrical contact appears, the rectification may arise from the different charge injection barriers for positive or negative sample polarity (as above) or from asymmetric alignment of energy levels across the molecule. To elaborate, we follow the discussion of Williams and co-workers [24] who outlined a simple model of molecular rectification, where a single electroactive unit was positioned asymmetrically with respect to electrodes and the HOMO and LUMO were positioned asymmetrically with respect to the Fermi level. In FFBT, the required conditions for rectification by an asymmetric barrier and asymmetric arrangement of the electrode Fermi energy with respect to the HOMO and LUMO are well met, since the HOMO and LUMO are 5.75 and $2.84 \mathrm{eV}$ respectively, and the Au work function is $5.1 \mathrm{eV}$. The experimental geometry clearly yields asymmetric injection barriers at the two ends of the molecule. The tip coated with molecules acts as one electrode since the molecules are attached by chemical bonding. The other end of the molecule is not chemically bound to the gold substrate and such an arrangement gives an asymmetric injection barrier at the two ends of the molecule. All the requisite conditions for molecular rectification [24] are thus met. Our demonstration of rectification by the FFBT molecules is a confirmation of this effect.

We finally estimate the number of molecules in the junction with the tip and surface in contact. The self assembled films form compact molecular layers. The Tour wire in particular is a short and rigid molecule and the self-assembled film is expected to be mechan- ically stiff. Accordingly, we assume an elastic contact at the junction. For a rounded tip of curvature $\sim 10 \mathrm{~nm}$ the radius of the tip-sample interaction area will be $\sim 1 \mathrm{~nm}$ for an elastic contact [25]. The spacing between molecules in the self assembled films is $\sim 0.5 \mathrm{~nm}$ and thus $\sim 10$ molecules or less would exist in the contact zone and contribute to the measured current.

In conclusion, we present a simple technique to investigate nanojunctions with a finite number of molecules, by self-assembling them on a gold coated STM tip. The STS measurement were conducted at room temperature, which is considered as rather challenging for other traditional methods $[2,3]$ using STM tips to locate the molecules self-assembled onto the gold surface. Results are presented for a Tour wire and a new molecule (FFBT) exhibiting rectification. The results demonstrate that the technique indeed measures the I$\mathrm{V}$ properties of the molecules to ensure good mechanical contact by appropriate setting of the tip-sample separation. The method should be considered as a qualitative approach only.

\section{References}

[1] A. Aviram and M. A. Ratner, Chem. Phys. Lett. 29, 277 (1974). http://dx.doi.org/10.1016/ 0009-2614 (74) 85031-1

[2] C. Joachim, J. K. Gimzewski and A. Aviram, Nature 408, 541 (2000). http://dx.doi.org/10.1038/ 35046000

[3] J. Chen, M. A. Reed, A. M. Rawlett and J. M. Tour, Science 286, 1550 (1999). http://dx.doi.org/ 10.1126/science. 286.5444.1550

[4] C. M. Fisher, M. Burghard, S. Roth and K. Klitzing, Appl. Phys. Lett. 66, 3331 (1995). http://dx. doi.org/10.1063/1.113747

[5] C. P. Collier, E. W. Wong, M. Belohradsky, F. M. Raymo, J. F. Stoddart, P. J. Kuekes, R. S. Williams and J. R. Heath, Science 285, 391 (1999). http://dx. doi.org/10.1126/science.285.5426.391

[6] M. A. Reed, J. Chen, A. M. Rawlett, D. W. Price and J. M. Tour, Appl. Phys. Lett. 78, 3735 (2001). http:// dx.doi.org/10.1063/1.1377042

[7] A. Vilam, J. Ghabboun and D. Cahen, J. Phys. Chem. B 107, 6360 (2003). http://dx.doi.org/10. 1021/jp026779b

[8] A. Vilan, C. Pejoux and D. Cahen, Adv. Funct. Mater. 17, 795 (2002). http://dx.doi.org/10.1002/adfm. 200290009

[9] L. Jones and J. M. Tour, Polym. Prepr. 36, 233 (1995).

[10] S. Datta, W. D. Tian, S. H. Hong, R. Reifenberger, J. I. Henderson and C. P. Kubiak, Phys. Rev. Lett. 79, 2530 (1997). http://dx.doi.org/10.1103/ PhysRevLett. 79.2530

[11] K. W. Hipps, Science 294, 536 (2001). http://dx.doi. org/10.1126/science. 1065708 
[12] X. D. Cui, A. Primak, X. Zarate, J. Tomfohr, O. F. Sankey, A. L. Moore, T. A. Moore, D. Gust, G. Harris and S. M. Lindsay, Science 294, 571 (2001). http:// dx.doi.org/10.1126/science. 1064354

[13] J. Reichert, R. Ochs, D. Beckmann, H. B. Weber, M. Mayor and H. V. L?hneysen, Phys. Rev. Lett. 88, 176804 (2002). http://dx.doi.org/10. 1103/PhysRevLett. 88.176804

[14] N. B. Zhitenev, A. Erbe and Z. Bao, Phys. Rev. Lett. 92, 186805 (2004). http://dx.doi.org/10. 1103/PhysRevLett. 92.186805

[15] U. Durig, O. Zuger, B. Michel, L. Haussling and H. Ringsdorf, Phys. Rev. B 48, 1711 (1993). http://dx. doi.org/10.1103/PhysRevB.48.1711

[16] J. M. Tour, L. Jones, D. L. Pearson, J. J. S. Lamba, T. P. Burgin, G. M. Whitesides, D. L. Allara, A. N. Parikh and S. V. Atre, J. Am. Chem. Soc. 117, 9529 (1995). http://dx.doi.org/10.1021/ja00142a021

[17] A. Salomon, D. Cahen, S. Lindsay, J. Tomfohr, V. B. Engelkes and C. D. Frisbie, Adv. Mat. 15, 1881 (2003). http://dx.doi.org/10.1002/adma. 200306091
[18] A. Nitzan, Annu. Rev. Phys. Chem. 52, 681 (2001). http://dx.doi.org/10.1146/annurev . physchem. 52 . 1.681

[19] V. Mujica, M. Kemp, A. Roitberg and M. A. Ratner, J. Chem. Phys. 104, 7296 (1996). http://dx.doi.org/ 10.1063/1.471396

[20] N. B. Zhitemev, H. Meng and Z. Bao, Phys. Rev. Lett. 88, 226801 (2002). http://dx.doi.org/10. 1103/PhysRevLett. 88.226801

[21] M. V. M. Rao, Ya. K. Su, T. S. Huang and Y.-C. Chen, Nano-Micro Lett. 2, 242 (2010). http://dx.doi.org/ $10.5101 / \mathrm{nml} \cdot \mathrm{v} 2 \mathrm{i} 4 \cdot \mathrm{p} 242-246$

[22] K. Stokbro and J. Taylor, J. Am. Chem. Soc. 125, 3674 (2003). http://dx.doi.org/10.1021/ja028229x

[23] A. Troisi and M. A. Ratner, J. Am. Chem. Soc. 124, 14528 (2002). http://dx.doi.org/10.1021/ ja028281t

[24] P. E. Kornilovitch, A. M. Bratkovsky and R. S. Williams, Phys. Rev. B 66, 165436 (2002). http:// dx.doi.org/10.1103/PhysRevB.66.165436

[25] K. L. Johnson, Contact Mechanics (Cambridge University Press, Cambridge, 1985). 